(4) D. V. Zagrebin, 'Method of improvement of Brown's Tables for the amendment of the Lunar ephemeris', Astr. J. U.S.S.R. 30, I, 1953.

(5) M. B. Shelesniak and E. A. Mitrofanova, 'Tables for the reduction of the lunar ephemeris to ephemeris time', Bull. Inst. Theor. Astr. 5, no. 1o/73, 1954.

(6) D. V. Zagrebin, E. A. Mitrofanova and G. M. Posniak, 'Difference between Ephemeris Time and Universal Time from observations of occultations of stars by the Moon', Bull. Inst. Theor. Astr. 6, no. I/75, I955.

(7) I. D. Yongolovich, 'On the history of the creation of nautical and air almanacs in our country', Bull. Inst. Theor. Astr. 5, no. 8/7 I, 498-5I I, I953.

(8) Bull. Inst. Theor. Astr. 5, no. ro/73, I954.

(9) I. D. Yongolovich, 'External gravitational field of the Earth and fundamental constants connected with it', Mem. Inst. Theor. Astr. 3, I-125, 1952.

(xо) H. Nowacki, 'Verbesserung von Ort und Eigenbewegung von 6I Cygni', Veröff. ARI, Heidelberg, no. 4, 1952.

(II) Trans. I.A.U. 7, 6I, r950.

(12) F. Link et al., Bull. Inst. Astr. Czechosl. 3, 76, 1952; 4, 170, 1953.

(I3) F. Link, B.A.C., sous presse.

(I4) F. Jink, Circ. I.A.U. no. I450, 1954.

\title{
Report of Meetings of Commission 4
}

President: Mr D. H. Sadler.

Secretary: Dr J. G. Porter.

At the first meeting on Tuesday, 30 August, sixty-two members were present. The President reported the loss to the Commission by death of Prof. J. Chazy and Admiral W. Benitez e Inglott.

In a discussion on the proposed definition of the unit of time, which had been adopted by the Comité International des Poids et Mesures at its meeting in September I954 (Trans. 9, p. 45I), Dr Clemence and Dr Gondolatsch pointed out the advantages of defining the unit in terms of the tropical year, rather than the sidereal year. As the matter had been referred to Commission 3I, which was not scheduled to meet until the end of the week, it was agreed that the President should notify the Executive Committee that Commission 4 would support the proposal if it were adopted by Commission $3 \mathrm{I}$.

The President then presented the following Report from the Directors of the National Ephemerides on the redistribution of astronomical calculations.

The conformity of the American Ephemeris and the (British) Nautical Almanac will involve a number of minor changes in the distribution of calculations between the two Offices; the principal one is that from I960 onwards the (British) Nautical Almanac Office will assume responsibility for the calculation of elements of lunar occultations.

The availability of reproducible material for the unified Almanac has led to the decision of the Astronomisches Rechen-Institut at Heidelberg to suspend publication, as from 1958, of the Astronomisch-Geodatisches Jahrbuch and to use the Nautical Almanac and later the unified Almanac instead. This in turn has made it possible for the Astronomisches RechenInstitut to take over, from the (British) Nautical Almanac Office, the publication, as from I960, of the international volume Apparent Places of Fundamental Stars. It is particularly appropriate that the A.R.-I., which is primarily responsible for the fundamental star places, should now assume responsibility for the apparent places based on those star positions. Most of the redistribution of calculations is directed towards the more efficient production of the international volume by the centralization of the calculation of apparent places of stars in a small number of Offices. 
Ephemeris Office
A.E. (Washington)
N.A. (Herstmonceux)
A.A. (Leningrad)
A.N. (San Fernando)
C.T. (Paris)

A.-G.J. (Heidelberg)

\section{At present}

calculates

162 Io-day stars

2 I6 Io-day stars

210 Io-day stars

378 Io-day stars

$32 \mathrm{FK}_{3}$ circumpolar stars

560 ro-day stars

$20 \mathrm{FK}_{3}$ circumpolar stars
Will in future

calculate

$$
\begin{gathered}
- \\
- \\
67 \text { additional } \\
\text { circumpolar stars } \\
\text { All } 52 \mathrm{FK}_{3} \\
\text { circumpolar stars } \\
\text { All I 483 FK } 3 \\
\text { Io-day stars }
\end{gathered}
$$

The Office of the American Ephemeris will take over from the Astronomisches RechenInstitut the calculation of those ephemerides of the satellites of Saturn which are at present made there; it will thus be responsible for all the satellite data in the Ephemerides, with the exception of the four Galilean satellites of Jupiter, which will continue to be calculated by the Office of the Connaissance des Temps.

The above redistribution will have the effect of centralizing the calculations for the Almanacs in a few Offices and those for the Apparent Places of Fundamental Stars in a few others. The Directors regard both publications as forming part of the complete 'astronomical ephemeris', and agree to regard contributions to either part as contributions to the whole. In some cases also, other calculations (such as the ephemerides of minor planets), which do not contribute directly to the published data in the national ephemerides, are being undertaken; these also should be regarded as contributions to the common task of astronomical calculation.

Dr Fricke and Prof. Subbotin spoke of the advantages of the new arrangements, and the Report was approved by the meeting.

There was considerable discussion on the proposal to omit the list of mean places of stars as from I958 from the volume Apparent Places of Fundamental Stars. The President and Dr Fricke explained the technical and financial difficulties involved in producing the list, but Dr Atkinson and Dr Randić asked for its retention for the use of observers and for teaching purposes. The President stated that H.M. Nautical Almanac Office was prepared, if necessary, to continue the list in 1958 and 1959; and Dr Fricke suggested that subsequently the list could be given every tenth year, using the FK 4 places and commencing, possibly, in rg6r. Prof. Subbotin and Prof. Zverev suggested that Commission 8 might be consulted, and it was agreed, on the suggestion of Dr Clemence, that the matter be postponed until Commission $8 \mathrm{had}$ considered it.

The proposal to give continuous tabulation of apparent places of ro-day stars at intervals of ro transits was agreed without discussion.

The addition of 67 extra circumpolar stars to the list of apparent places as from rg6o was considered, and Dr Fricke explained why no supplementary stars of lower declinations were thought desirable. He agreed with Dr Atkinson that these 67 stars should be clearly marked as being additional to the $\mathrm{FK}_{3}$ stars, and proposed to give them in a separate list.

After a brief discussion it was agreed to adopt, in the national ephemerides as from I 960 , day numbers referred to the equinox of the nearest beginning of the year.

Two proposals made by Dr Clemence were agreed without discussion:

(a) that, as from 1960, the orbit of Jupiter V should be that of van Woerkom (Astr. Pap., Wash., I3, part I);

(b) that, as from 1960, the period of rotation of Mars should be that of Ashbrook (Astr. J. 58, p. 145, 1953).

Dr Atkinson spoke on the inconsistency between the adopted constant I/297 for the Earth's flattening and Brown's value of $3422^{\prime \prime} .54$ for the constant term in the sine of the 
Moon's equatorial horizontal parallax, which is based on an assumed flattening of I/294. In the course of the discussion which followed, Dr Clemence agreed that there were inconsistencies in Brown's Tables, but said that this unsatisfactory position could not be improved until a new lunar theory became available. It was always possible for individuals to apply their own corrections, and the Offices of the national ephemerides would be willing to supply observers with special values of the geocentric quantities $S$ and $C$ to suit any value of the flattening. After some further discussion, it was agreed that no change should be made in the published values as used in the national ephemerides.

Dr Atkinson drew attention to the different values of the semi-diameters of the Sun and Moon used in the ephemerides, and pointed out the requirements in eclipse work of a mean semi-diameter of the Moon to which limb corrections could be applied. Dr Clemence stated that this matter had been considered, and the U.S. Nautical Almanac Office would use any value agreed upon by Commission 4 ; but since predictions were made eight years in advance, he would require due notice of any change. Discussion was deferred until the next meeting.

On Wednesday, 3I August, there were fifty-four members present. The President reported that Commission 8 had discussed the inclusion of mean places in the volume Apparent Places of Fundamental Stars and had agreed that mean places should be included in full for the years 1958 and 1959 , but that, to simplify preparation of copy, constellation names should be omitted. For I 960 and subsequent years (when the volume will be prepared by the Astronomisches Rechen-Institut) it was agreed that mean places be printed every tenth year. For the first year of publication it had been decided that the list should be based on $\mathrm{FK}_{4}$ and be given for equinox $1960 \cdot 0$, even though it may be I96I or 1962 before it can be published. These decisions were approved by the meeting.

Discussion continued on the question of the Moon's semi-diameter, and it was felt that Watts' limb corrections would eventually lead to a better value of this quantity. Dr Atkinson said that his sole object was to select one value which could be used in both lunar ephemeris and in eclipse work. Dr Clemence suggested that a decision be deferred until a value could be fixed to which Watts' corrections could be applied. After some further discussion the President suggested that Dr Atkinson and Dr Clemence should exchange views on this matter and make definite proposals at the next meeting in I958. This was agreed.

The President then referred to the semi-diameter of the Sun, for which a value of I6 $6^{\prime}$ or". 8 at mean distance was proposed for the $0^{\text {h }}$ ephemeris in the unified Almanac as from I96o. This enhanced value has been used for many years in the Nautical Almanac, but has now been criticized by Dr Atkinson, who would prefer to have the true value used. Dr Clemence pointed out that the tabulated value was used mainly by meridian observers, and asked what value would satisfy modern meridian observations. Mr Symms stated that the Nautical Almanac values show no systematic differences from observations.

After some discussion, the President proposed that the present value of $16^{\prime}$ or." 18 be retained. Dr Clemence asked if the other national ephemerides would use the same value as the unified Almanac, and affirmative replies were given by Prof. Subbotin, Dr Fricke, Capt. de la Puente, Prof. Danjon and Dr Fayet.

The President announced that Commission I 7 had agreed that the occultation programme should be continued unchanged for several years. He also reported that the Improved Lunar Ephemeris had now been published and copies had been sent to most observatories.

On the proposal of Prof. Danjon, the Draft Report was formally adopted.

Mrs Gossner showed a new form of eclipse chart to be used in the unified Almanac, and the President showed photostat specimens of the revised form of Abridged Nautical Almanac to be introduced in 1958 .

The question of the definition of the unit of time remaining outstanding, Prof. Danjon remarked that the resolution adopted at the Rome meeting in 1952 required no other change than the substitution of 'tropical' for 'sidereal'. Dr Clemence then proposed that Commission 4 should adopt the change definitively, and this was agreed unanimously. 